\title{
Report on the 45th Annual Meeting of the Society for Neuroscience in Anesthesiology and Critical Care, 2017
}

\author{
Barkha Bindu ${ }^{1}$ Hemanshu Prabhakar ${ }^{1}$ \\ ${ }^{1}$ Department of Neuroanaesthesiology and Critical Care, Neurosciences \\ Centre, All India Institute of Medical Sciences, New Delhi, India
}

J Neuroanaesthesiol Crit Care 2018;5:45-47.

The 45th Annual Meeting of the Society for Neuroscience in Anesthesiology and Critical Care (SNACC) was held at the Westin Waterfront Hotel, Boston, Massachusetts, United States from October 19 to 20, 2017. The city of Boston, with its historical relevance to anesthesia, was the ideal host for anesthesiology meeting of international standards. The theme of this year's meeting was Brains and Machines and was jointly provided by the American Society of Anesthesiologists (ASA) and the SNACC. This 2-day meeting witnessed 350 attendees from across the world. The organizing committee of the 45th Annual Meeting laid out a very comprehensive scientific schedule that highlighted latest innovations and advances in the fields of neuroanesthesia and neurosciences. The scientific schedule progressed smoothly from workshops to basic and clinical sciences on day 1 and from general sessions to neurosciences sessions on day 2 .

The official meeting of SNACC commenced on Thursday afternoon, the 19th of October. Three workshops were conducted concurrently with a basic science symposium and a clinical science symposium followed by a dinner symposium. The Ultrasound in OR and ICU workshop was moderated by Drs. Wael M. Ali SakrEsa, David Anthony, Hesham Elsharkawy, and Ehab S. Farag, and it was extremely clinically oriented and practical in approach. The workshop provided attendees with excellent hands-on experience of various central venous and arterial cannulation sites and respective ultrasonographic views. Delegates also had the opportunity to try their hands at lung ultrasound and intracranial pressure assessment by optic nerve sheath ultrasonography. Participants also had first-hand experience of various views of transthoracic echocardiography and assessment of volume responsiveness based on inferior vena cava diameter. Ultrasound for gastric content was a new and interesting aspect of the workshop that discussed identification of full stomach by ultrasound and estimation of volume of gastric contents. The workshop on Simulation in Neurosciences conducted offsite at Boston Children's Hospital was moderated by Drs. Michael L. James, Marco A. Maurtua, Colleen M. Moran, and Elizabeth H. Sinz. The simulation workshop discussed the
Address for correspondence Barkha Bindu, MD, DNB, Department of Neuroanaesthesiology and Critical Care, Neurosciences Centre, All India Institute of Medical Sciences, New Delhi 110029, India (e-mail: barkhabindu@gmail.com).

goals and advantages of simulation training, aspects of adult learning, technical aspects of simulation, designing scenarios, resource management, and simulation with mannequin. The third workshop was on Career Development. It was moderated by Drs. William M. Armstead, PhD, and Chanannait Paisansathan. Dr. Robert Tamburro discussed the National Institute of Health Funding Opportunities and Challenges. This was followed by an interactive discussion by a panel that included Drs. William M. Armstead, Chanannait Paisansathan, and Robert Tamburro.

This year's basic science symposium was on Fluid Therapy and was moderated by Dr. William M. Armstead, PhD. The first lecturer, Dr. Michael J. Souter, professor of anesthesiology and pain medicine at the University of Washington, reviewed body fluid homeostasis and discussed the various types of fluids available and their physiological effects in neurologically injured patients. He discussed the special challenges and reasons why homeostasis is impaired in neurologically injured patients and that external correction requires detailed assessment of these perturbations. The following lecture by Dr. Fitz Roy E. Curry, PhD, discussed the Revised Starling Principle of transvascular fluid exchange. He emphasized that the classical Starling principle is far from correct. According to Revised Starling Principle, fluid retention depends on filtration rate that can be altered by vasoconstriction, and not on oncotic pressure differences between plasma and interstitial fluid. The last lecture by Dr. Martin Smith from United Kingdom reviewed the role of lactate as an energy substrate in traumatic brain injury (TBI) and the importance of functional mitochondria in this context. He also discussed emerging evidence regarding the role of lactate supplementation and its potential to reduce the incidence of cognitive impairment after TBI.

The clinical science symposium on Neuromonitoring was moderated by Drs. Linda S. Aglio and Antoun Koht. The first lecture was by Dr. Stacie G. Deiner on depth of anesthesia. Dr. Deiner discussed the recommendations made by different societies regarding monitoring of depth of anesthesia. Ongoing trials and various resources available for
DOI https://doi.org/ $10.1055 / \mathrm{s}-0037-1618325$ ISSN 2348-0548.
Copyright $\odot 2018$ Indian Society of Neuroanaesthesiology and Critical Care
License terms

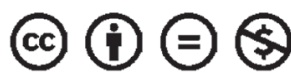


electroencephalography (EEG) training of anesthesia practitioners were also reviewed. The following lecture was by Dr. Laura B Hemmer on "cranial nerve monitoring." Dr. Hemmer is associate professor of anesthesiology and neurological surgery at the Northwestern University of Feinberg School of Medicine, Chicago. She discussed the technique of cranial nerve monitoring and the clinical relevance of various responses obtained during monitoring. She also discussed the anesthetic concerns and the utility of special tracheal tubes that have prior integrated electrodes. She highlighted the utility of the lateral spread response in specific situations. Dr. Antoun Koht, Chicago, lectured on anesthetic management for sensory evoked potential/motor evoked potential (SSEP/MEP) monitoring and discussed, in a very interesting manner, the approach to intraoperative changes in evoked potentials. He also elaborated on the clinical significance of central conduction time. The last lecture was by Dr. Ben A. Palanca on EEG. Dr. Palanca talked about nonconvulsive status epilepticus and burst suppression. Various postoperative complications associated with burst suppression were also highlighted.

This years' dinner symposium focused on Perioperative Monitoring of the Brain and was moderated by Dr. Deepak Sharma. The reception and dinner provided the participants with an opportunity to interact with each other. After SNACC President Dr. George A. Mashour welcomed the attendees, Dr. Deepak Sharma introduced the session and the speakers. The first speaker, Dr. Jamie W. Sleigh, University of Auckland, New Zealand, discussed the need for a standard monitor that can reliably monitor the anesthetized brain. He elaborated on the pitfalls and limitations of existing EEG-based monitors and the need for developing algorithms that can better represent the anesthetic state of the brain. There were further lectures by Dr. George A. Mashour on EEG in the conscious and the anesthetized brain. Dr. Jan Classen lectured on the role of EEG in comatose patients.

The second day of the meeting started with breakfast followed by committee meetings. The mobile meeting guide (MMG) served as an excellent resource for attendees who could access scientific information related to the meeting on their phones. Scientific schedule of the second day started with Maurice Albin Keynote Lecture that was moderated by Dr. Piyush M. Patel. After Dr. George A. Mashour welcomed the attendees, Dr. Ole Isacson lectured on neuroregeneration. Dr. Isacson beautifully described the process of neuroregeneration in some invertebrates and how neuroscience is evolving basing on knowledge gained from these organisms. He described a few patients of Parkinson's disease who received transplantation of dopamine neurons in their brains and the outcomes in them. Dr. Isacson highlighted that neuron transplantation into human brain might be the way out for some neurodegenerative diseases. The lecture was followed by some very interesting questions from the attendees.

The pre-lunch mini symposium was themed Brains and Machines and was moderated by Dr. Andrew Kofke, past president of SNACC. The first lecture was by Dr. Ali Rezai on Restorative Neurosurgery. He discussed the emerging role of deep brain stimulation (DBS) in TBI and Alzheimer's disease. He also discussed less invasive interventions, such as focused ultrasound treatment and neural bypass technology and their potential role in neurosurgery. This was followed by a very interesting lecture by Dr. Jeffrey G. Ojemann on Brain-Machine Interface. Dr. Ojemann is a pediatric neurosurgeon at the University of Washington. He discussed the use of electrocorticographic (ECoG) arrays for functional mapping of human sensorimotor cortex and learning new motor tasks. The last lecture was by Dr. Cindy Chestek, $\mathrm{PhD}$, an engineering graduate. She lectured on the use of brain-computer interfaces models to study general anesthesia. She impressed the audience with some very interesting experiments performed on monkeys at her institute in this context.

After a short break for refreshments, the first poster session commenced. A total of 143 posters from 14 different countries were presented. Both poster sessions were conducted through electronic posters displayed on nine monitors. Posters were also accessible via the MMG throughout the meeting. The MMG provided the participants with an opportunity to view as well as post questions about posters to the authors. The wide variety of neuroscience research presented as posters at the meeting provided a glimpse of the vastness of research being performed across the globe. The first poster session was followed by the business lunch. There was a special lunch session also this year that ran concurrently with the business lunch in a different room. This special lunch session included a quiz that was called Neuroanesthesiology Review Fun that was played very excitedly by the participants through their mobile phones. This session was moderated by Drs. Chanannait Paisansathan and Shobana Rajan and was targeted mainly at residents and trainees. Questions were based on basics of neuroanesthesia, anticoagulants, and spine surgeries. Two prestigious books, Handbook of Neuroanesthesia edited by Dr. Cottrell and Complications in Neuroanesthesia edited by Dr. Hemanshu Prabhakar, were given away as prizes to the winners. The second poster session commenced after lunch and closed with refreshments.

The second mini symposium of second day was on Perioperative Coagulation and Anticoagulation and was moderated by Dr. Dhanesh K. Gupta. The first speaker, Dr. Louanne M. Carabini, Northwestern Memorial Hospital, talked about Novel Anticoagulants. He reviewed the pharmacology and dosing of newer direct acting oral anticoagulants (DAOC) and enumerated the evidence available regarding indications for their usage. He also discussed the tests to be used for monitoring their activity. The last speaker of this session was Dr. Jerrold Levy, professor at Duke University School of Medicine, who spoke extensively about perioperative management of newer anticoagulants. Dr. Levy elaborated on the specific antidotes available for the newer anticoagulants and emphasized that the perioperative management of these agents should be based on procedure-related bleeding risk. He clarified that perioperative bridging while interrupting DAOC does not provide any therapeutic benefit and can cause increased major bleeding. 
The New Science Session this year discussed the GOLIATH (General or Local Anesthesia in Intra Arterial Therapy) and SIESTA (Sedation versus Intubation for Endovascular Stroke TreAtment) trials regarding endovascular treatment of ischemic stroke. This session was moderated by Dr. Deepak Sharma. Dr. Mads Rasmussen, associate professor from Denmark, who was involved in conducting the GOLIATH trial updated the audience regarding other latest trials in this field. He also informed about the investigators' plans to combine and analyze the data from three recent trials. In response to points strongly raised by the audience about the GOLIATH trial, Dr. Levy clarified that some of the results of the GOLIATH trial are about to be published shortly and will help clear confusion regarding certain aspects of this trial.

The last session of this year's meeting was the Plenary Session moderated by Dr. George A. Mashour. Dr. John D. Hartung, PhD, associate editor of the prestigious Journal of Neurosurgical Anesthesiology so far, gave a very interesting and engaging talk on how to read and do neuroscience research. He brought out the loopholes in the currently existing system of conducting research that enable false research results to be published. Dr. Hartung suggested that establishing a common manuscript submission portal for all journals will help check this problem to some extent. At the end of Dr. Hartungs' lecture, Dr. Martin Smith was announced as the new editor of the Journal of Neurosurgical Anesthesiology. Dr. Jeffrey J. Pasternak, the new president of SNACC, then gave the closing remarks, and the meeting was finally concluded with wine and cheese and a meeting of Special Interest Groups.

The Annual Meeting of 2018 is scheduled to be held at San Francisco, California in the month of October next year. The beauty of San Francisco and the exuberant scientific contents of the SNACC annual meeting make a highly desired combination to attract participants from all parts of the globe. And given the high standards of knowledge that SNACC has always maintained, the next meeting too is bound to be amazing.

\section{Funding}

None.

\section{Conflict of Interest}

None. 